\title{
Interleukin-17 Stimulates STAT3-Mediated Endothelial Cell Activation for Neutrophil Recruitment
}

\author{
Shaopeng Yuan ${ }^{\mathrm{a}}$ Shimeng Zhang ${ }^{\mathrm{b}}$ Yuanyuan Zhuang ${ }^{\mathrm{a}}$ He Zhang ${ }^{\mathrm{b}}$ Jinye Baia \\ Qi Hou \\ aeijing Key Laboratory of New Drug Mechanisms and Pharmacological Evaluation Study, Institute \\ of Materia Medica, Chinese Academy of Medical Sciences \& Peking Union Medical College, Beijing, \\ bDepartment of Radiation Toxicology and Oncology, Beijing Institute of Radiation Medicine, Beijing, \\ China
}

\section{Key Words}

Interleukin-17 • Endothelial cells activation - Neutrophil recruitment • Signal transducers and activators of transcription $3 \cdot$ Chemokine

\begin{abstract}
Background/Aims: Interleukin-17 (IL-17) is a major pro-inflammatory cytokine that initiates and maintains inflammation. However, the molecular mechanisms as to how IL-17 influences endothelial cells to promote neutrophil recruitment are not fully understood. Methods: Human endothelial cells (HMECs) were stimulated with IL-17, and investigated for proliferation, migration, and tubule formation activities. Transwell chemotaxis and adhesion assays were performed to assess neutrophil recruitment. Cytokine production was measured by Cytokine Array Chip and ELISA. Western blotting and immunofluorescent analysis were used to detect the phosphorylation and translocation of STAT3. Specific inhibitors, small interfering RNA, and phosphorylation mutants were used to confirm that IL-17 induced STAT3 activation via IL-17RA signaling. Results: Activation of HMECs with IL-17 induced STAT3 phosphorylation and nuclear translocation, which were associated with induction of GRO- $\alpha, G M-C S F$ and IL- 8 , and neutrophil recruitment. Phosphorylation of STAT3 was identified mainly at the tyrosine in position 705 (Y705), and the Y705F mutants attenuated IL-17-mediated STAT3 activation. Moreover, specific inhibitors, FLLL31, or siRNA silencing of STAT3 attenuated HMECs activation, resulting in inhibition of GRO- $\alpha$, GM-CSF, IL-8 production, and neutrophil recruitment. Furthermore, phosphorylation of STAT3 was identified as downstream of IL-17RA signaling. Conclusions: IL-17 induced STAT3 activation as a necessary step in endothelial cell activation and neutrophil recruitment.
\end{abstract}




\section{Cellular Physiology Cell Physiol Biochem 2015;36:2340-2356 \begin{tabular}{l|l} 
and Biochemistry Published online: July 27, 2015 & $\begin{array}{l}\text { C 2015 S. Karger AG, Basel } \\
\text { www.karger.com/cpb }\end{array}$ \\
\hline
\end{tabular} \\ Yuan et al.: IL-17 Stimulates STAT3-Mediated Endothelial Cell Activation}

\section{Introduction}

Interleukin-17 (IL-17), mainly produced by CD4 T-helper 17 (Th17) cells, is a major pro-inflammatory cytokine that initiates and maintains inflammation $[1,2]$. Besides Th17 cells, several other cells are capable of producing IL-17, including macrophages, dendritic cells, natural killer cells, natural killer T cells, lymphoid tissue inducer cells, and $\gamma \delta$-T cells. Previous studies in both mice and humans have shown that IL-17 plays a pivotal role in promoting multiple inflammatory diseases including rheumatoid arthritis, multiple sclerosis, asthma, chronic obstructive pulmonary disease (COPD), and cancer [3-6].

The broad impact of IL-17 is due to its ability to induce the chemotaxis and accumulation of inflammatory cells, such as neutrophils, to the sites of inflammation [7, 8]. However, IL-17 does not directly promote the chemotaxis of human neutrophils from peripheral blood [9]; rather, it promotes the release of recruitment factors from the inflamed microenvironment. Recent studies demonstrate that IL-17 induces the production of IL-6, IL-8, Gro- $\alpha$, G-CSF, and CCL20 in epithelial cells, synovial fibroblasts, colonic myofibroblasts, and airway smooth muscle cells [10-14]. Moreover, the induction of these inflammatory factors promotes recruitment and accumulation of inflammatory cells, such as neutrophils [15].

In addition to inflammatory cells, endothelial cells play a crucial role in the inflammation process [16]. During acute and chronic inflammation, immune responses stimulate endothelial cell activation and induce angiogenesis [17]. Activated endothelial cells produce a series of adhesion factors, as well as cytokines and chemokines, to recruit inflammatory cells, such as neutrophils and monocytes [18]. Recently, the role of IL-17 in this process has been described in the context of tumorigenesis and rheumatoid arthritis [19-22]. Some key mediators induced by IL-17 include: CXCR2, G-CSF, IL-6, VEGF, and PDGF. Thus, these data suggest that IL-17 promotes the activation of endothelial cells and the induction of angiogenesis. However, the molecular mechanisms underlying the function of IL-17 in these processes are still being defined.

The IL-17 family of cytokines contains six members: IL-17, IL-17B, IL-17C, IL-17D, IL$17 F$, and IL-25. IL-17 and IL-17F can form homo- or hetero-dimers that bind a heteromeric receptor complex containing IL-17RA and/or IL-17RC $[2,23,24]$. Binding of IL-17 to the IL-17 receptors typically leads to activation of the NF- $\kappa B$, MAPKs, ERK, or C/EBPs signaling pathways [25]. Recently, Act1 was identified as an important mediator via its interaction with IL-17R through TRAF6-dependent and -independent downstream pathways [26]. In addition, IL-17-mediated activation of STAT3 has been reported in airway epithelial cells [27] and smooth muscle cells $[23,28]$. However, the requirement of STAT3 in IL-17-activated endothelial cells has not been fully explored.

STAT3 is a member of the STAT family of proteins, which are important latent regulators during the inflammation process $[29,30]$. Activation of STAT3 involves a tyrosine phosphorylation cascade involving recruitment by non-receptor tyrosine kinases including the JAK family (Tyk2, Jak1, 2, 3). Recruitment of STAT3 to the receptor kinase complex occurs via the interaction of its SH2 domain with a phosphorylated tyrosine residue at the receptor or kinase complex. STAT3 is then phosphorylated at a single tyrosine residue in the C-terminal domain, which leads to its dimerization, nuclear translocation, and ultimately the transcription of inflammatory factors [31]. Induction of inflammatory factors, including IL8, has been linked to the regulation of STAT3 activation [32]. In addition, the differentiation and regulation of Th17 cells, macrophages, eosinophils, and neutrophils are also associated with STAT3 signaling. More importantly, STAT3 plays pivotal roles in the regulation of angiogenesis and endothelial cell activation [33]. Thus, STAT3 is a major mediator in regulating inflammation, immune cell development, and endothelial cell activation. Therefore, understanding the potential connection between IL-17 signaling and STAT3 activation in regulating endothelial cell activation will help to fully elucidate the molecular mechanisms of IL-17 in inflamed microenvironments.

In this study, we used a model of human endothelial cells to examine the function of STAT3 in IL-17-mediated endothelial cell regulation and inflammatory cell recruitment. 


\section{Cellular Physiology Cell Physiol Biochem 2015;36:2340-2356 \begin{tabular}{l|l|l}
\cline { 2 - 2 } DOI: 10.1159/000430197 & C $2015 \mathrm{~S}$. Karger AG, Basel
\end{tabular} and Biochemistry Published online: July 27, 2015

IL-17 induction leads to the activation of endothelial cell proliferation, migration, and tubule formation. In addition, IL-17 promoted the induction of GRO- $\alpha$, GM-CSF, and IL-8 to regulate neutrophil recruitment, while silencing of STAT3 through siRNAs retarded these processes. Further investigations revealed that tyrosine phosphorylation of STAT3 play an important role in regulating this process. Mutation of the phosphorylation site Y705 directly attenuated STAT3 nuclear translocation and transcriptional activity in the presence of IL-17. In addition, a specific STAT3-Y705 inhibitor, FLLL31 [34], reduced the expression of GM-CSF and IL-8, resulting in attenuation of neutrophil recruitment and endothelial cell activation. Further investigation identified that inhibition of IL-17RA reduced phosphorylation at Y705. Taken together, our results demonstrate the ability of IL-17 to regulate the production of inflammatory factors for neutrophil recruitment via the STAT3 signaling pathway.

\section{Materials and Methods}

\section{Cell Culture}

HMEC is a human dermal microvascular endothelial cell line (HDMEC, Sciencell, Carlsbad, CA) and was cultured at $37^{\circ} \mathrm{C}, 5 \% \mathrm{CO}_{2}$ in Dulbecco's modified Eagle's medium (DMEM) with $10 \%$ fetal calf serum (FCS, HyClone, Logan, UT) and 100 units/ml Penicillin/Streptomycin. HL-60 cells are cultured in RPMI1640 medium with $10 \%$ FCS, if not specified.

\section{Materials}

The JAK2 inhibitor, AG490, was purchased from Caliochem (Gibbstown, NJ). The STAT3 inhibitor, FLLL31, and other materials were purchased from Sigma-Aldrich Chemical (St. Louis, MO) unless specified otherwise. ELISA kits were purchased from eBioscience (San Diego, CA). Recombinant human IL-17, bFGF, and TNF- $\alpha$ were obtained from R\&D Systems (Minneapolis, MN). Commercial SignalSilence ${ }^{\circ}$ STAT3 siRNA and Control siRNA were purchased from Cell Signaling Technology (Beverly, MA).

\section{Cell proliferation assay}

The proliferation of HMECs was assessed with the Cell Counting Kit-8 (CCK-8, Dojindo, Kumomoto, Japan) according to the manufacturer's instructions. Briefly, HMECs were seeded into 96-well plates (1 $\times 10^{4}$ cells/well), and following serum deprivation for 24 hours, then were stimulated with IL-17 (1-100 $\mathrm{ng} / \mathrm{ml})$ for 24 or 48 hours, respectively. TNF- $\alpha(10 \mathrm{ng} / \mathrm{ml})$ was used as a positive control for proliferation induction. CCK-8 at a final concentration of $5 \mathrm{mg} / \mathrm{ml}$ was then added to the culture, and cell proliferation was measured 3 hours later using a microplate reader (OD value at $450 \mathrm{~nm}$ ). Data are represented as relative fold increase over untreated control groups.

To examine the inhibitors of STAT3 signaling pathways, cells were pretreated with inhibitors of JAK2 (AG490) or STAT3 (FLLL31) for 1 hour prior to IL-17 treatment (50 ng/ml). Cell proliferation was measured 48 hours later using CCK-8 as described above.

\section{Tubule formation assay}

Tubule formation assays were conducted as previously described [35]. Briefly, 24-well plates were pre-coated with $100 \mu \mathrm{l} /$ well matrigel for 1 hour at $37^{\circ} \mathrm{C}$. HMECs were then seeded at $1 \times 10^{4}$ cells/well and incubated with bFGF (10 ng/ml) or different concentrations of IL-17. After 6 hours of incubation, cells were fixed and visualized by bright-field microscopy. Tubule formation images were captured with the Olympus DP1T digital camera system (Olympus Optical, Tokyo, Japan) and assessed in six randomly selected fields per well. To examine the functions of STAT3 signaling pathway inhibitors, the cells were pretreated with AG490 $(10 \mu \mathrm{M})$ and FLLL31 $(10 \mu \mathrm{M})$ for 1 hour before IL-17 treatment, and then examined as described above.

\section{Migration assay}

HMECs were plated into 12-well plates. After the cells formed a monolayer, a "wound" was created by scrapping a straight line into the bottom of the well with a p200 pipette tip. The wells were then washed in PBS and cultured in DMEM including IL-17 plus 2\% FCS for 4 hours. Following this incubation, HMECs were 


\section{Cellular Physiology Cell Physiol Biochem 2015;36:2340-2356 \begin{tabular}{l|l|l} 
DOI: 10.1159/000430197 & (C) 2015 S. Karger AG, Basel
\end{tabular} and Biochemistry Published online: July 27, 2015 \\ Yuan et al.: IL-17 Stimulates STAT3-Mediated Endothelial Cell Activation}

fixed in $1 \%$ gluteraldehyde, stained with $0.1 \%$ crystal violet, and viewed by light microscopy (magnification $\times 20$ ). Images of cells in six randomly selected fields per well were captured by the Olympus DP1T digital camera system (Olympus Optical). Relative migratory activities were calculated by comparing the width difference of IL-17-treated HMECs with that of the untreated control group.

\section{Immunofluorescence labeling}

HMECs were plated into 6-well plates and pre-treated with vehicle (0.1\% DMSO), FLLL31 (10 $\mu$ M), or AG490 $(10 \mu \mathrm{M})$ for 30 minutes. The cells were then stimulated with IL-17 (50 ng/ml) for 1 hour. Treated cells were washed with ice-cold PBS, fixed with cold methanol, and cultured in blocking buffer for 30 minutes. Following blocking, the cells were washed with PBS and cultured with an anti-STAT3 monoclonal antibody (Cell Signaling Technology) overnight at $4^{\circ} \mathrm{C}$. After washing, the cells were labeled with a FITC-conjugated anti-mouse secondary antibody, stained with DAPI, and viewed with a scanning confocal imaging system. Six random fields in each well (magnification $\times 60$, Leica DMI 6000B-CS) were chosen for analysis.

To examine the nuclear translocation of STAT3 and its dominant-negative mutants after IL-17 treatment, the GFP-fused wild type and Y705F mutants of STAT3 were transfected for 24 hours into HMECs with Lipofectin according to the manufacturer's instructions. Then HMECs were treated with IL-17 (50 ng/ $\mathrm{ml}$ ) for 2 hours. The cells were viewed by fluorescence confocal microscopy and six random fields in each well were counted.

\section{Western blotting analysis of STAT3 translocation into nuclei}

HMECs were serum-starved in DMEM for 24 hours and then treated with IL-17 (50 ng/ml) for 0.5-2 hours. Cytosolic and nuclear proteins were then obtained using the nuclear-cytosol extraction kit (Applygen, Beijing) with a protease inhibitor cocktail (Roche), according to the manufacturer's instructions. Equal amounts of protein from individual samples were resolved by electrophoresis in a SDS polyacrylamide gel, and gel slices were then transferred onto polyvinylidene difluoride membranes (Millipore, Bedford, MA). The membranes were washed with Tris-buffered saline plus $0.1 \%$ Tween- 20 (TBST), and then blocked with $5 \%$ nonfat milk for 1 hour at room temperature. The membranes were then probed with anti-phosphoTyr705 (Y705), anti-phospho-Ser727 (S727) of STAT3, and anti-STAT3 monoclonal antibodies overnight at $4^{\circ} \mathrm{C}$ (Cell Signaling Technology). Lamin B and $\beta$-actin antibodies were used to indicate nuclear and cytosolic protein controls, respectively. Following overnight incubation, membranes were washed extensively with TBST and developed by chemiluminescence detection with a LAS3000-mini image analysis system.

\section{RNA interference assay}

HMECs were transfected with inference siRNAs targeting STAT3, IL-17RA, or IL-17RC with GenoFect transfection reagents (Genobio, Beijing, China) for 24 hours according to the manufacturer's instructions. The cells were then cultured in the presence of IL-17 (50 ng/ml) and collected at the time points indicated. Commercial SignalSilence $囚$ STAT3 siRNA and Control siRNA were purchased from Cell Signaling Technology. Specific 27mer siRNA duplexes targeting IL-17RA and IL-17RC were purchased from OriGene Technologies (Rockville, MD). The duplex sequences are as following:

IL-17RA: rGrGrGrArUrArCrCrArArGrArUrArArArUrUrGrCrArUrGCG

IL-17RC: rCrUrCrArArArGrArCrGrArUrGrUrGrCrUrArCrUrGrUrUrGG

\section{STAT3 luciferase activity assay}

The construction of the STAT3 recognition promoter motif in the pGL6 vector was previously described $[36,37]$. Stably transfected HMEC cells were treated with IL-17 with or without chemical inhibitors in serum-free medium for 4 hours. The cells were then harvested and lysed according to the luciferase reporter assay kit protocol (Promega, Madison, WI). Cell lysates were measured using a luminometer (Promega) according to the manufacturer's instructions. Relative luciferase activities were normalized to those of untreated control groups.

\section{Neutrophils and endothelial cell adhesion assay}

Isolations of human neutrophils from whole blood were performed with solution of Polymorphprep® according to the manufacturer's instructions (AXIS-SHIELD, Oslo, Norway). The final centrifugation of isolated human neutrophils was adjusted to $2 \times 10^{6} / \mathrm{ml}$. The neutrophils were then added above the HMEC 


\section{Cellular Physiology Cell Physiol Biochem 2015;36:2340-2356 \begin{tabular}{ll|l} 
and Biochemistry & $\begin{array}{l}\text { DOI 10.1159/000430197 } \\
\text { Published online: July 27, } 2015\end{array}$ & $\begin{array}{l}\text { O 2015 S. Karger AG, Basel } \\
\text { www.karger.com/cpb }\end{array}$ \\
\cline { 2 - 3 }
\end{tabular} \\ Yuan et al.: IL-17 Stimulates STAT3-Mediated Endothelial Cell Activation}

monolayer to investigate the adhesion and migration activities. Briefly, HMEC cells were seeded into 96well plates and pre-incubated with or without FLLL31 or AG490 for 30 minutes followed by treatment with IL-17 (50 ng/ml) for 6 hours. Following incubation, HMECs were washed with PBS and cultured in serumfree medium. Isolated human neutrophils were pre-labeled with MTT ( $2 \times 10^{5} /$ well $)$ and added above the HMEC monolayer for 2 hours. Next, the supernatants and unbound neutrophils were discarded, and HMECs were washed with PBS and dissolved in DMSO for 10 minutes. The OD value at $570 \mathrm{~nm}$ was then assessed using a microplate reader (BioTek, Winooski, VT). Adhesion activities are indicated as the relative OD 570 difference of IL-17-treated cells with that of untreated controls.

Adhesion assays of HL-60 cells were performed using the method described above. Briefly, HMEC cells were seeded into 96-well plates and pre-incubated with or without FLLL31 or AG490 for 30 minutes followed by treatment with IL-17 $(50 \mathrm{ng} / \mathrm{ml})$ for 6 hours. Then, HL- 60 cells pre-labeled with MTT $\left(1 \times 10^{6} /\right.$ $\mathrm{ml}$ ) were added to the medium of HMECs for 2 hours, and the OD value at $570 \mathrm{~nm}$ was measured.

\section{HL-60 chemotaxis and transmigration assay}

Transwell chemotaxis assays were carried out to assess HL-60 recruitment to endothelial cells using the 3- $\mu \mathrm{m}$ Millicell inserts (Millipore) [38]. Briefly, HMECs were seeded into 24-well plates and grown until confluent. The cells were then pre-incubated with or without FLLL31 or AG490 for 30 minutes, and then treated with IL-17 (50 ng/ml) for 6 hours. HL-60 cells stained with CellTracker Green $\left(2 \times 10^{6} / \mathrm{ml}\right)$ were then added to the top chambers above the HMEC monolayer, and allowed to migrate. After 2 hours, the cells under the chambers were fixed with 4\% formaldehyde in PBS for 15 minutes. HL-60 cells that had migrated to the bottom chamber of HMECs were viewed by fluorescence confocal microscopy. Five random fields in each well were selected for analysis.

\section{Human Cytokine Antibody Array Chip Assay}

HMECs were serum-starved overnight, then cultured in the presence of IL-17 (50 ng/ml) for 24 hours. The culture medium was collected and centrifuged at 12,000 g for $10 \mathrm{~min}$. Inflammatory factors in the supernatant were then analyzed using a Cytokine Antibody Array Chip (Cat: AAH-CYT-3-8, RayBio), according to the manufacturer's instructions. Analysis was performed in triplicate. Data are represented as the fold increase of IL-17-treated groups over control groups.

Statistical analysis

Statistical significance was analyzed using the student's t-test. $P$ values $<0.05$ were considered statistically significant. All data are representative of at least three separate experiments.

\section{Results}

\section{IL-17 promotes endothelial cell activation in vitro}

First, we examined whether IL-17 promoted the proliferation of endothelial cells. HMECs were treated in vitro with different concentrations of IL-17 and the Cell Counting Kit8 was used to assess proliferation rate. After treatment for 24 or 48 hours, IL-17 promoted endothelial cell proliferation in a concentration-dependent manner (Fig. 1A). Compared with the untreated control group, concentrations of both 50 and $100 \mathrm{ng} / \mathrm{ml}$ IL-17 treatment significantly promoted endothelial cell proliferation $(P<0.01)$.

Next, we examined the ability of IL-17 to induce tubule formation on endothelial cells grown in vitro. HMECs were cultured on Matrigel and treated with IL-17 for 6 hours, after which tubule formation was subsequently captured and assessed. Similar to the positive control (10\% FCS), IL-17 significantly promoted endothelial tubule formation in HMECs (Fig. 1B and 1C). The observed increase of tubule formation indicated that IL-17 activation might also increase HMEC migration. Therefore we used a "wound healing assay" to assess HMEC migration. HMECs monolayers were disrupted using a pipette tip to create a "wound". The migration of HMECs into the "wound" was then assessed 4 hours later. Results showed that IL-17 promoted the migration of HMECs in a concentration-dependent manner (Fig. 1D and 1E). In addition, pre-treating HMECs with an IL-17R blocking antibody prevented IL- 


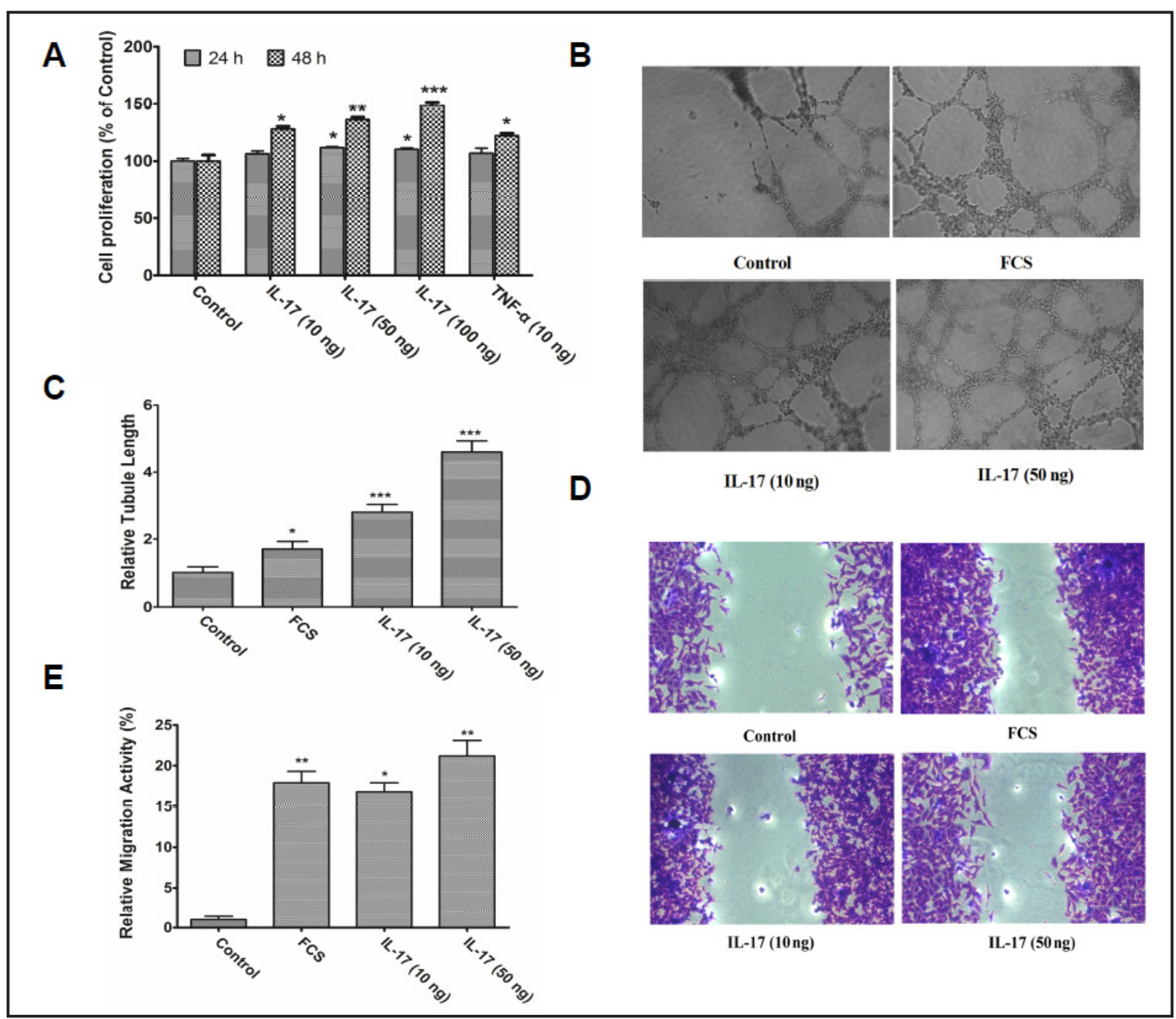

Fig. 1. IL-17 promotes endothelial cell activation in vitro. A. HMECs were stimulated with IL-17 $(10,50,100$ $\mathrm{ng} / \mathrm{ml}$ ) for 24 or 48 hours, at which point proliferation was assessed using the Cell Counting Kit-8. TNF- $\alpha$ $(10 \mathrm{ng} / \mathrm{ml})$ was used as a positive control. Data are represented as the relative fold induction over untreated control groups. *, $P<0.05$ vs. Control; ${ }^{* *}, P<0.01$ vs. Control; ${ }^{* *}, P<0.001$ vs. Control. B. HMECs were plated in Matrigel and treated with 10\% FCS, bFGF (10 ng/ml), IL-17 (10 and $50 \mathrm{ng} / \mathrm{ml}$ ). Tubule formation was assessed in six randomly selected fields per well, and the images were captured with the Olympus DP1T digital camera system (Olympus Optical). C. Tubule images were captured, and lengths were assessed from six randomly selected fields per well. ${ }^{*}, P<0.05$ vs. Control group; ${ }^{* *}, P<0.01$ vs. Control; ${ }^{* * *}, P<0.001$ vs. Control. D. Following exposure to IL-17, HMECs were fixed in $1 \%$ gluteraldehyde, stained with $0.1 \%$ crystal violet, and viewed by light microscopy (magnification $\times 20$ ). FCS $(10 \%)$ treatment as the positive control. The images were captured from six randomly selected fields per well. E. Relative migratory activities of the different groups were calculated by the scraped width difference of endothelial cells per well compared with that of the untreated control group. The images were captured from six randomly selected fields per well.

17-mediated migration (Data not shown). These data confirmed that IL-17 promotes HMEC migration. Collectively, these data support the role of IL-17 in promoting endothelial cell activation.

IL-17 promotes endothelial cell production of inflammatory factors to recruit neutrophils

Since endothelial cells can promote the recruitment of neutrophils to sites of inflammation, we investigated whether IL-17 promotes neutrophil recruitment to HMECs. Niggli and colleagues reported that differentiated HL-60 cells, a human neutrophil cell line, were valid for investigating neutrophil migration and chemotaxis [39]. The results showed that there were little differences between differentiated HL-60 cells and human neutrophils with 
A

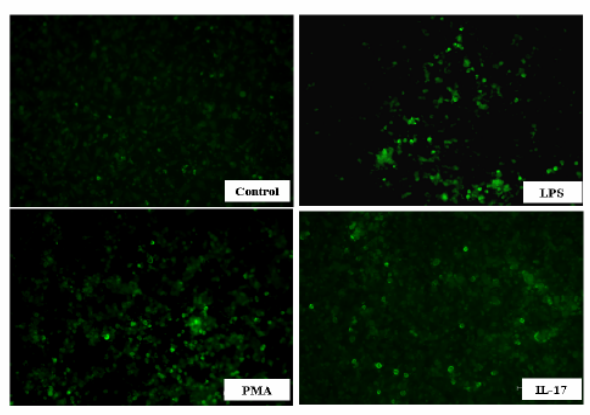

B

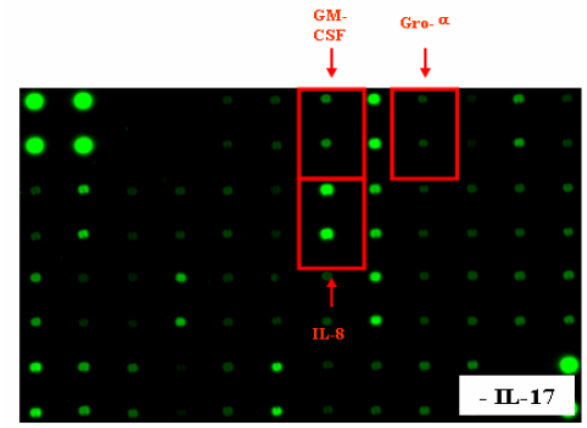

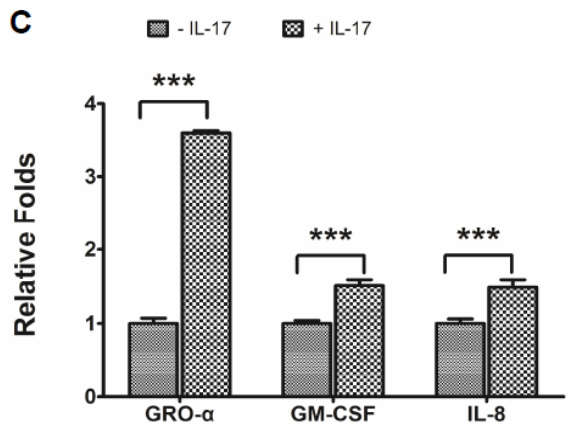

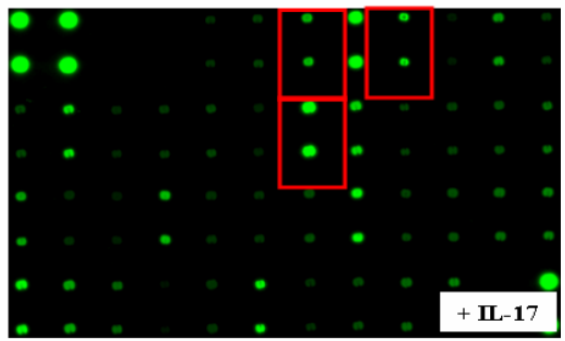

Fig. 2. IL-17 promotes inflammatory factor production to recruit neutrophils to HMECs. A. HMECs were seeded into the bottom chambers of trans-well plates and then treated as follows: LPS $(1 \mu \mathrm{g} / \mathrm{ml})$, PMA (100 $\mathrm{nM})$, and IL-17 (50 ng/ml) for 6 hours. CellTracker Green stained HL-60 cells $\left(2 \times 10^{6} / \mathrm{ml}\right)$ were then added to the top chambers and allowed to migrate. After 2 hours, HL-60 cells in the bottom wells fixed with $4 \%$ formaldehyde and viewed by fluorescence confocal microscopy. The photos showed as 5 random fields in each well. B. Serum-starved HMECs were treated with IL-17 (50 ng/ml). After 24 hours, the cell culture media was collected and centrifuged at 12,000 g for 10 minutes. Cytokines were then analyzed using a RayBio Cytokine Antibody Array Chip. Each group was analyzed in triplicate. Inflammatory factors that were significantly altered are outlined in red. C. Statistical relative fold induction of the inflammatory factors to the control groups. Each group was analyzed in triplicate. Data are represented as the fold induction relative to the control groups.

regards to the chemotactic stimuli effects on morphology, chemokinesis, and chemotaxis. We thus used HL-60 to investigate the effects of IL-17-induced inflammatory cells recruitment. HMECs were pre-treated with IL-17, and then HL-60 cells stained with CellTracker Green were added to the top chambers of the transwell plates and allowed to migrate to HMECs for 2 hours. Results showed that IL-17-induced activation of HMECs significantly increased the migration of HL-60 cells to endothelial cells (Fig. 2A). As previously mentioned, IL-17 can promote structural cells like epithelial cells to produce inflammatory factors. We therefore wondered whether IL-17 induced the production of pro-inflammatory factors from HMECs to regulate HL-60 recruitment. To this end, we examined the expression of inflammatory factors from IL-17-treated culture medium using Cytokine Antibody Array Chips. Treatment of HMECs with IL-17 for 24 hours mainly elevated the expression of Gro- $\alpha$, GM-CSF, and IL-8 among the 42 detected factors $(P<0.001$ with control group, Fig. $2 \mathrm{~B}$, and $2 \mathrm{C})$. Gro- $\alpha$, GM-CSF, and IL-8 play important roles in regulating neutrophil recruitment and survival, thus these data suggest that IL-17 induces production of pro-inflammatory factors from endothelial cells to regulate recruitment of neutrophils during the inflammation process.

STAT3 is crucial in regulating IL-17-induced endothelial cell activation

As mentioned above, STAT3 is associated with chronic inflammation, endothelial cell activation, and the transcription of inflammatory factors. Cytokine activation promotes 


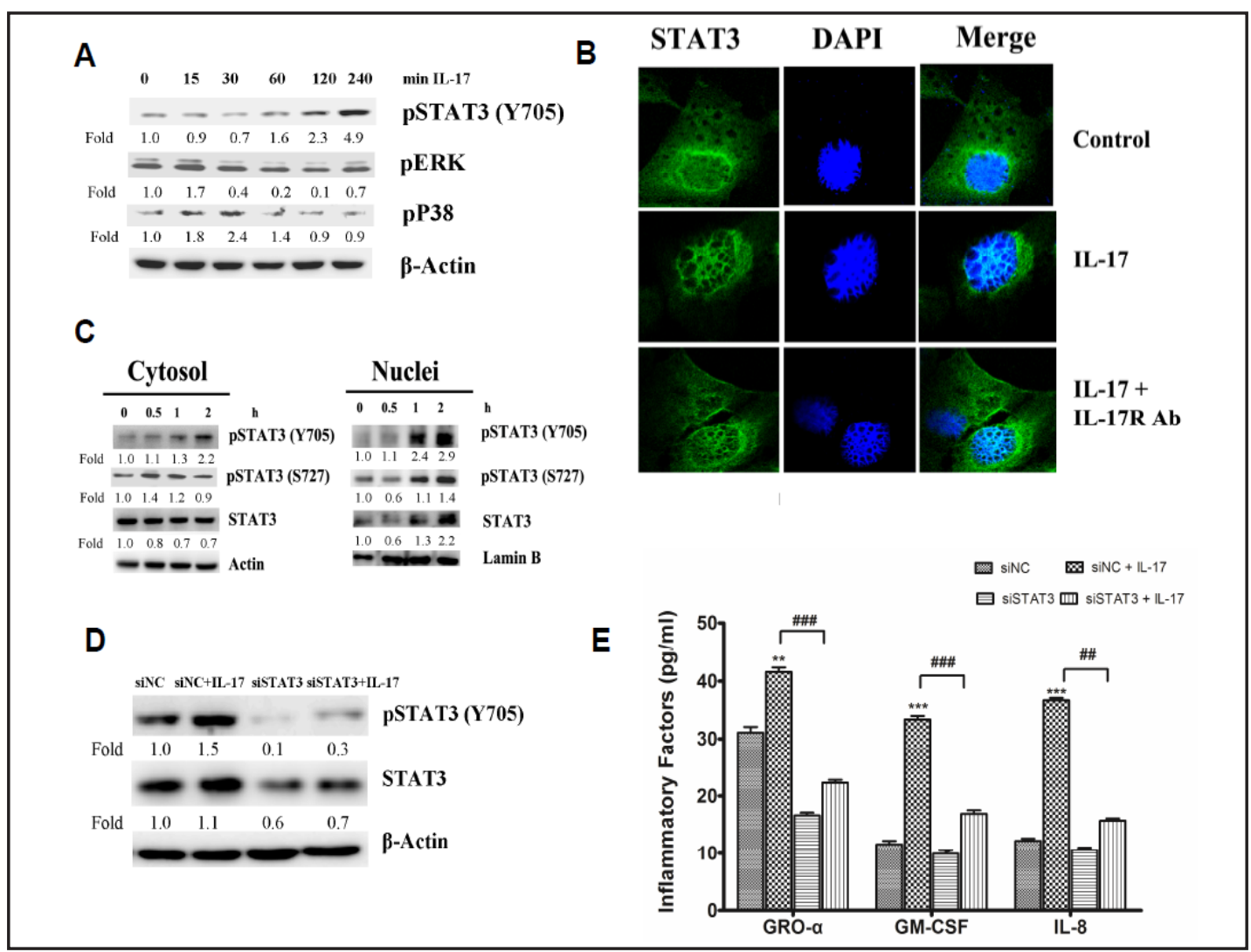

Fig. 3. IL-17-mediated endothelial cell activation requires STAT3. A. HMECs were serum-starved for 24 hours, and then treated with IL-17 (50 ng/ml). After 0.5-4 hours the cells were collected and analyzed by western blot using p-STAT3 (Y705), pERK, and pP38 monoclonal antibodies, respectively. The blots were also probed with anti- $\beta$-actin antibodies as the internal control. Fold indicated the calculated relative intensity of target protein/ $\beta$-actin by densitometry. B. HMECs were pre-treated with or without anti-IL-17R antibody for 1 hour and then activated with IL-17. The cells were then subjected to immunofluorescence labeling with an anti-STAT3 monoclonal antibody. Green represents STAT3 labeling, and blue represents the nuclei stained with DAPI (60× objective lens). Scale bar: $10 \mu \mathrm{m}$. C. HMECs were serum-starved for 24 hours, and then treated with IL-17 (50 ng/ml). After 0.5-2 hours the cells were collected, and lysed with the nuclear-cytosol extraction kit. The samples were then analyzed by Western blot using p-STAT3 (Y705, S727) and STAT3 monoclonal antibodies. The blots were also probed with anti- $\beta$-actin and anti-Lamin B antibodies as internal controls. Fold indicates the calculated relative intensity of target protein/ $\beta$-actin by densitometry. D. HMECs were transfected with inference siRNAs targeting STAT3, or a random sequence of siRNA. After 24 hours, the cells were treated with IL-17 (50 ng/ml) for 2 hours. The cells were then collected and lysed, and p-STAT3 (Y705) and STAT3 were analyzed by western blot. Fold indicates the calculated relative intensity of target protein/ $\beta$-actin by densitometry. E. HMECs were transfected with inference siRNAs targeting STAT3, or a random sequence of siRNA. After 24 hours, the cells were treated with IL-17 (50 ng/ml) for 6 hours. The culture medium was then collected and ELISA kits were used to evaluate the levels of GRO- $\alpha$, GM-CSF, and IL-8. * $P<0.05$ vs. Control; ** $P<0.01$ vs. Control; ${ }^{* * *}, P<0.001$ vs. Control. \#\#, $P<0.01$ vs. IL-17 treatment groups; \#\#, $P<0.001$ vs. IL-17 treatment groups.

STAT3 phosphorylation, dimerization, and nuclear translocation, which collectively promote gene transcription. The amino acid residues Tyr705 (Y705) and Ser727 (S727) are important phosphorylation sites of STAT3. To investigate whether STAT3 phosphorylation is required for IL-17-induced activation of STAT3, we evaluated the phosphorylation status of these residues upon IL-17 treatment. Western blot analysis revealed that IL-17 promoted Y705 phosphorylation of STAT3 when cells were treated with IL-17 (Fig. 3A). On the other hand, S727 phosphorylation was only detected at early time points (Data not shown). In KARGER 
addition, treatment of IL-17 elevated the phosphorylation and activation of ERK and p38 at the early time points treatment, while no significant changes were observed in later time points, as previously reported [40]. Next, the immunofluorescence analysis revealed that IL-17 induced STAT3 nuclear translocation (Fig. 3B). Comparatively, STAT3 in untreated groups was almost located exclusively in the cytosol. To further confirm the role of IL-17, HMECs were pre-treated with an IL-17R blocking antibody. Results showed that the nuclear translocation of STAT3 was blocked by anti-IL-17R antibody pre-treatment. Further western blot analysis revealed that IL-17 promoted the tyrosine phosphorylation of STAT3 both in the cytosolic and nuclear fractions. However, serine phosphorylation was only detected at significant levels in the nuclear fractions (Fig. 3C). These results confirm that IL-17 promotes the activation of STAT3 in endothelial cells.

To further evaluate the role of STAT3 in this process, we used siRNAs to attenuate the expression of STAT3 in HMECs. Western blotting analysis revealed that commercial siRNAs against STAT3 significantly attenuated the protein expression level of STAT3, including tyrosine phosphorylation of STAT3 following IL-17 treatment (Fig. 3D). In addition, knockdown of STAT3 decreased the expression of Gro- $\alpha$, GM-CSF, and IL-8 following exposure to IL-17 (Fig. 3E). Interestingly, these results indicated STAT3 might regulate the induction of Gro- $\alpha$, GM-CSF, and IL-8.

Phosphorylation of STAT3 705 tyrosine plays an important role in IL-17-induced endothelial cell activation

Given that IL-17 promoted the phosphorylation and nuclear translocation of STAT3, we next investigated mechanisms of STAT3 activation in this process. We mutated the major tyrosine 705 phosphorylation site of STAT3 and constructed GFP-fused dominant-negative mutants (Y705F). Next, the dominant-negative mutants were transfected into HMECs and treated with IL-17. Immunofluorescent imaging revealed that after IL-17 treatment, nuclear translocation of STAT3 was blocked in the Y705F mutant-transfected HMECs (Fig. 4A). These results confirmed that the Y705F mutant retarded the tyrosine phosphorylation of STAT3, and attenuated its translocation to the nuclei. Since nuclei-associated STAT3 modulates the transcription and expression of target genes, we sought to establish whether these phosphorylation sites were important for IL-17-mediated STAT3 activation. Therefore, we fused a luciferase reporter plasmid to a STAT3 recognition motif, transfected the recombinant plasmid into HMECs, and measured the effect of IL-17 treatment on STAT3 regulation of the reporter gene. Consistent with our immunofluorescence analysis, IL-17 treatment elevated the luciferase activity of STAT3 compared to the untreated controls. As expected, the Y705F mutation prevented IL-17-induced transcriptional activity of STAT3 in HMECs (Fig. 4B). Therefore, these results indicate that phosphorylation of Y705 is necessary for IL17-mediated STAT3 activation in HMECs.

To further evaluate the role of Y705 in IL-17-mediated endothelial cell activation, the specific inhibitor of tyrosine phosphorylation of STAT3 was used to investigate the function of IL-17 in HMECs. FLLL31 is a newly developed STAT3 inhibitor which selectively binds to Janus kinase 2 (JAK2) and the STAT3 Src homology-2 (SH2) domain, and specifically suppresses the Y705 phosphorylation of STAT3. Our results showed that in the presence of FLLL31, the proliferation function induced by IL-17 was attenuated (Fig. 4C). To further evaluate the role of tyrosine phosphorylation of Y705 in the promotion of endothelial cell activation, we examined the effects of FLLL31 on tubule formation. As shown in Fig. 4D and 4E, FLLL31 attenuated IL-17-mediated tubule formation in matrigel. These results further indicate that STAT3 is an important regulator in IL-17-mediated endothelial cell activation, and that phosphorylation of STAT3 at Y705 plays an important role in this process.

Phosphorylation of Y705 is relative to recruitment of neutrophils to HMECS

Since IL-17 promotes endothelial cell activation to recruit HL-60 (Fig. 2A), we next examined the role of Y705 phosphorylation of STAT3 on IL-17-activated HMECs in regulating neutrophil recruitment. HMECs were pre-treated with FLLL31 and then activated with IL- 


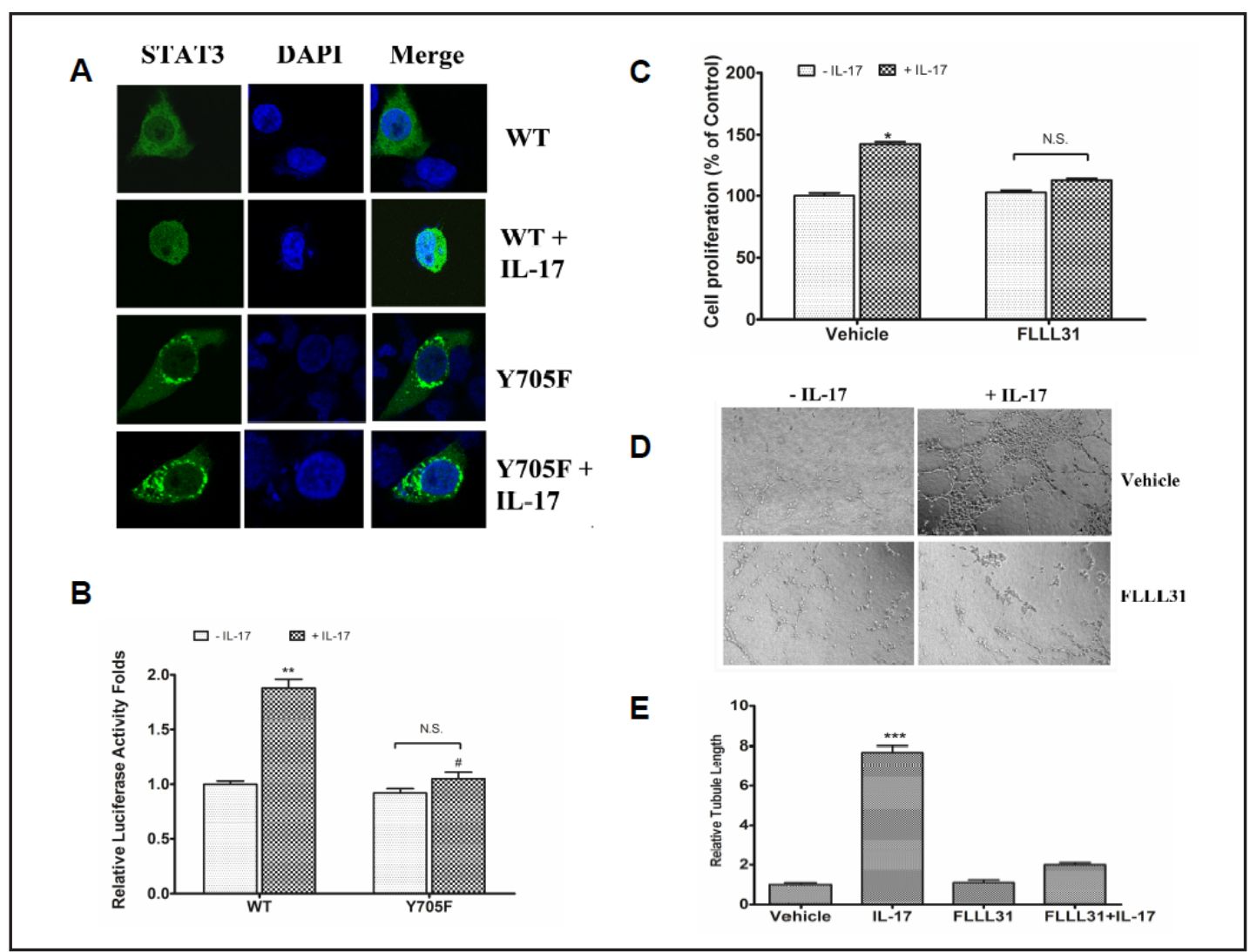

Fig. 4. Phosphorylation of STAT3 705 tyrosine plays an important role in IL-17-induced endothelial cell activation. A. GFP-fused wild type and phosphorylation dominant-negative (DN) mutants of STAT3 were transfected into HMECs for 24 hours. Following transfection, HMECs were treated with IL-17 (50 ng/ml) for 2 hours. The cells were then viewed by fluorescence confocal microscopy and five random fields in each well were selected for analysis. Green represents STAT3 labeling, and blue represents nuclei stained with DAPI. Scale bar: $10 \mu \mathrm{m}$. B. HMECs stably expressing STAT3 Luciferase were transfected with wild type or a phosphorylation DN mutant (Y705F) of STAT3 for 24 hours. The cells were then treated with IL-17 (50 ng/ $\mathrm{ml}$ ) for 4 hours and luciferase activity was analyzed. ${ }^{* *}, P<0.01 \mathrm{vs}$. Control; \#, $P<0.05$ vs. IL-17 treatment group. N.S. = Not Significant $(P>0.05$ vs. mutant alone). C. HMECs were pre-treated with or without FLLL31 for 1 hour, followed by exposure to IL-17 (50 ng/ml). After 48 hours, cell proliferation was measured using Cell Counting Kit-8. *, P <0.05 vs. Control. D. HMECs were plated in Matrigel and pre-treated with FLLL31 for 1 hour, and then incubated with IL-17 (50 ng/ml). Tubule formation was visualized 4 hours later by light microscopy and images were selected from six random fields per well. E. Statistical quantitation of tubule lengths was assessed from six randomly selected fields per well. ${ }^{* *}, P<0.001$ vs. Control.

17. Adhesion assay results showed that IL-17 treatment of HMECs significantly increased the adhesion of HL-60, and this process was attenuated when HMECs were pre-treated with FLLL31 and AG490 (Fig. 5A). To further confirm the effects of FLLL31 and AG490 on human neutrophil recruitment, we isolated circulating neutrophils from healthy donors and performed adhesion assays. As expected, our results showed that HMECs promoted recruitment and adhesion of neutrophils upon IL-17 treatment, which are similar as the observations in HL-60 cells. In addition, our results showed that pre-treatment with FLLL31 and AG490 could attenuate neutrophil recruitment to endothelial cells, which further confirmed that STAT3 plays important roles in the downstream signaling pathway (Fig. 5B).

Following neutrophils adhesion assays in response to IL-17, activated HMECs were assessed using transwell plates as mentioned above. HMEC monolayers were pre-incubated with or without FLLL31 for 30 minutes, and then treated with IL-17. After 6 hours, HL60 cells stained with CellTracker Green were added to the top chambers of the transwell

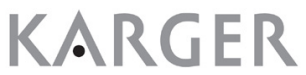


A

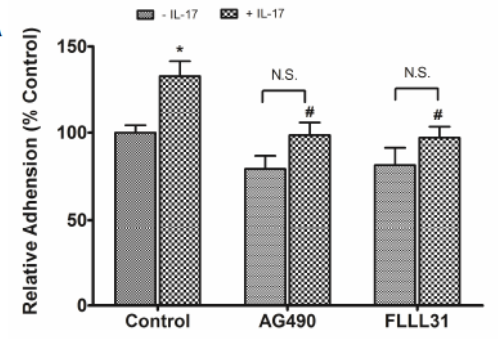

B

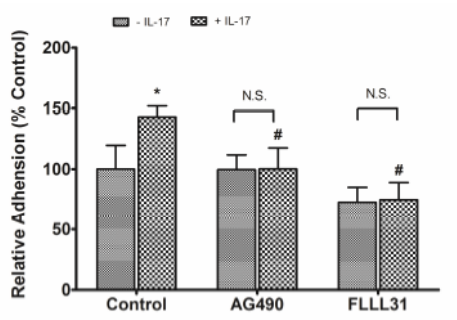

D

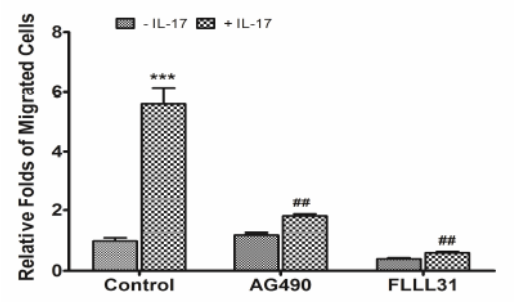

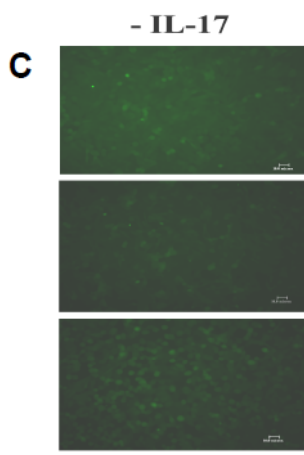

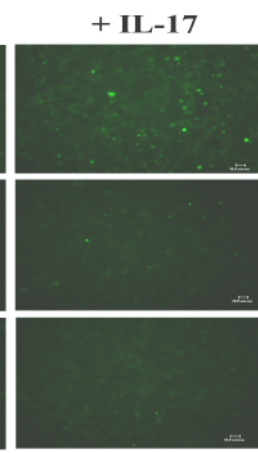

Control

E

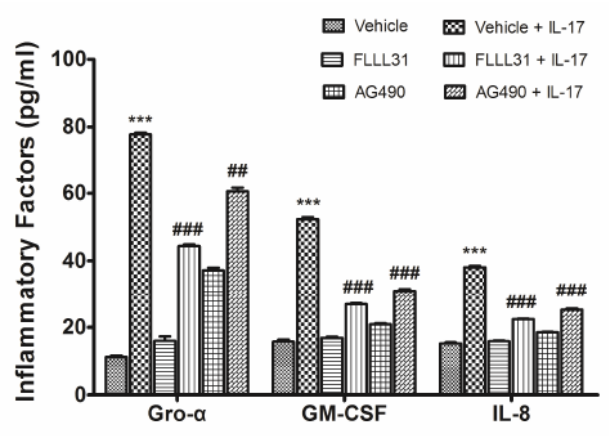

Fig. 5. Tyrosine Phosphorylation of STAT3 on Y705 is relative to recruitment of neutrophils to HMECs. A. HMEC cells were seeded into 96-well plates and pre-incubated with or without FLLL31 or AG490 for 30 minutes, and then treated with IL-17 (50 ng/ml) for 6 hours. The HMECs were then washed 3 times with PBS, and serum-free medium was added to the wells. MTT-labeled $\left(1 \times 10^{6} / \mathrm{ml}\right)$ HL-60 cells were then added to the wells. After 2 hours the supernatants were removed, the cells were washed 3 times with PBS, and dissolved with DMSO for 10 minutes. The OD value at $570 \mathrm{~nm}$ was assessed using a microplate reader. Data are represented as the relative activity compared to the untreated control. *, $P<0.05$ vs. Control; \#, $P<0.05$ vs. IL-17 treatment group. B. Adhesion assays of isolated human neutrophils from healthy donors were performed as described above. *, $P<0.05$ vs. Control; \#, $P<0.05$ vs. IL-17 treatment group. C. HMECs were seeded into the bottom chambers of trans-well plates and treated as mentioned above. CellTracker Green stained HL-60 cells $\left(2 \times 10^{6} / \mathrm{ml}\right)$ were then added to the top chambers and allowed to migrate. After 2 hours, the cells were fixed with $4 \%$ formaldehyde in PBS for 15 minutes, and HL-60 cells in the bottom wells were viewed by fluorescence confocal microscopy. Five random fields in each well were counted. D. Statistical analysis of the transmigrated HL-60 cells to the bottom endothelial cells. The average number of migrated HL-60 cells (Green) was determined from five random fields. ${ }^{* *}, P<0.001$ vs. Control. E. Expression of inflammatory factors in the presence of FLLL31 or AG490 on HMECs. HMECs were pre-incubated with or without FLLL31 or AG490 for 30 minutes and then treated with IL-17 (50 ng/ml) for 6 hours. The culture medium was then collected and ELISA kits were used to evaluate levels of GRO- $\alpha$, GM-CSF, and IL-8. *, $P<$ 0.05 vs. Control; **, $P<0.01$ vs. Control; ***, $P<0.001$ vs. Control. \#\#, $P<0.01$ vs. IL-17 treatment groups; \#\#\#, $P<0.001$ vs. IL-17 treatment groups. N.S. means Not Significant $(P>0.05$ vs. Control).

plates and allowed to migrate for 2 hours. As expected, IL-17-treatment activation of HMECs significantly induced the migration of HL-60 cells; however, this recruitment was inhibited by pre-treatment with FLLL31 (Fig. 5C and 5D). Interestingly, consistent with the STAT3 siRNA results, treatment with FLLL31 also significantly decreased the expression of Gro- $\alpha$, GM-CSF, and IL-8 in HMECs (Fig. 5E). Since these cytokines are major regulators in neutrophil recruitment and survival, our results indicate that IL-17 promotes the STAT3 activation on 


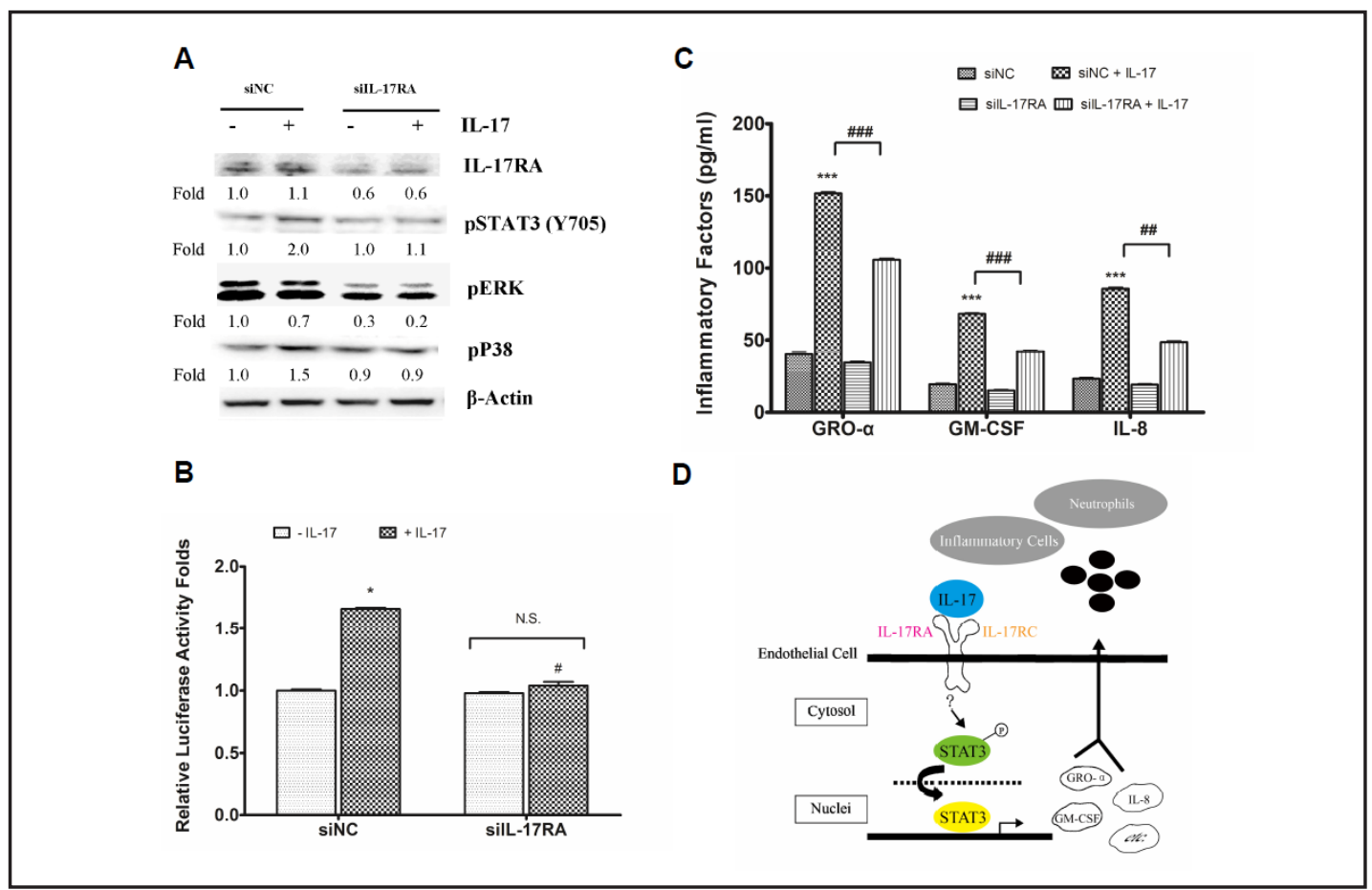

Fig. 6. IL-17RA is the major receptor promoting STAT3 regulation in endothelial cells. A. HMECs were transfected with siRNAs targeting IL-17RA or a random sequence for 24 hours, and then treated with IL-17 (50 $\mathrm{ng} / \mathrm{ml}$ ) for 4 hours. Then, cells were collected and analyzed by western blot using IL-17RA, p-STAT3 (Y705), pERK, and pP38 monoclonal antibodies. The blots were also probed with anti- $\beta$-actin antibodies as an internal control. Fold indicates the calculated relative intensity of target protein/ $\beta$-actin by densitometry. B. HMECs stably expressing STAT3 Luciferase were transfected with siRNAs targeting IL-17RA or a random sequence for 24 hours. The cells were then treated with IL-17 (50 ng/ml) for 4 hours and luciferase activity was measured. *, $P<0.05$ vs. Control; N.S.= Not Significant $(P>0.05$ vs. silL-17RA alone). C. HMECs were transfected with siRNAs targeting IL-17RA or a random sequence for 24 hours, and then treated with IL-17 $(50 \mathrm{ng} / \mathrm{ml})$ for 12 hours. The culture medium was then collected and ELISA kits were used to examine the levels of GRO- $\alpha$, GM-CSF, and IL-8. ${ }^{* * *}, P<0.001$ vs. Control; \#\#\#, $P<0.001$ vs. IL-17 treatment group. D. An abbreviated cartoon of the signaling pathway. IL-17 promotes the activation of the JAK-STAT3 pathway via IL-17RA, resulting in phosphorylation of tyrosine at position Tyr-705 (Y705) and nuclear translocation of STAT3. Activated endothelial cells produce the pro-inflammatory chemokines and cytokines, including Gro- $\alpha$, IL-8, and GM-CSF, which, in turn, promote neutrophil recruitment and adhesion to endothelial cells.

endothelial cells to produce inflammatory factors, which play vital roles in the recruitment of neutrophils. Also, these data suggest that tyrosine phosphorylation at Y705 in STAT3 is necessary for IL-17-mediated neutrophil recruitment by endothelial cells.

\section{IL-17-mediated STAT3 activation mainly occurs through IL-17RA}

Given that IL-17RA is the major receptor of IL-17, we wondered whether tyrosine phosphorylation of STAT3 is relative to IL-17RA. We then silenced IL-17RA in HMECs with a specific siRNA. Knockdown of IL-17RA attenuated the activation of Y705 phosphorylation on STAT3 (Fig. 6A). In addition, pre-treatment of IL-17RA monoclonal antibodies inhibited the tyrosine phosphorylation of STAT3 upon IL-17 treatment (Data not shown). Thus, the modulation of STAT3 is linked to IL-17RA. Next, we investigated the transcriptional activity of STAT3 after silencing IL-17RA in HMECs. As expected, silencing of IL-17RA prevented IL-17-induced transcriptional activity of STAT3 in HMECs (Fig. 6B). Moreover, silencing of IL-17RA significantly reduced the release of GRO- $\alpha$, GM-CSF, and IL-8 by HMECs (Fig. 6C). Therefore, our results support that IL-17, through IL-17RA, can regulate the STAT3 signaling KARGER 


\section{Cellular Physiology Cell Physiol Biochem 2015;36:2340-2356 \begin{tabular}{l|l} 
and Biochemistry Published online: July 27, 2015 & $\begin{array}{l}\text { C 2015 S. Karger AG, Basel } \\
\text { www.karger.com/cpb }\end{array}$ \\
\hline
\end{tabular} \\ Yuan et al.: IL-17 Stimulates STAT3-Mediated Endothelial Cell Activation}

pathway and the production of inflammatory factors, which might play important roles in the recruitment of neutrophils.

\section{Discussion}

In this study, we investigated the function and molecular mechanisms of IL-17mediated endothelial cell activation and neutrophil recruitment. Our results indicate that IL-17 directly activated endothelial cells through promoting proliferation, migration, and tubule formation. In addition, IL-17 promoted the secretion of the neutrophil recruitment factors GRO- $\alpha$, GM-CSF, and IL-8 from HMECs, which enhanced the adhesion and neutrophil recruitment to HMECs. Further experiments showed that these processes were directly correlated with to STAT3 regulation in HMECs. IL-17 promoted phosphorylation and nuclear translocation of STAT3, while silencing of STAT3 by RNA interference significantly attenuated IL-17-mediated endothelial cell activation, inflammatory factors secretion, and neutrophil recruitment. Moreover, tyrosine phosphorylation on Y705 was important for STAT3 regulation following IL-17 activation. Our results showed that dominant-negative mutant and specific inhibitors of Y705 phosphorylation attenuated the function of IL17 in HMECs. Furthermore, phosphorylation of Y705 could be partly attributed to the IL17RA transducing signal, since phosphorylation of Y705 could be inhibited by interference of IL-17RA. Based on these observations, we conclude that IL-17 promotes activation of endothelial cells through the STAT3 signaling pathway, which in turn enhances neutrophil recruitment (Fig. 6D).

In the present study, IL-17 directly promoted endothelial cell activation via migration and tubule formation. These results are consistent with previous observations using other endothelial cell lines [41-43]. IL-17 also promoted the proliferation of endothelial cells in a dose-dependent manner $(P<0.05)$, with higher doses of IL-17 increasing proliferation by approximately 50\%. Moreover, our data confirmed the effects of endothelial cells response to IL-17 on adhesion regulation and inflammatory cells recruitment. Although expression of adhesion molecules on HMECs was not examined during our investigations, ICAM-1 induction in response to IL-17 has been previously reported [11]. In addition, pre-treating HMECs with IL-17R blocking antibodies significantly attenuated IL-17-mediated HMEC activation. Collectively, these results establish IL-17 as an endothelial cell activation stimulus. Moreover, our data confirmed the effects of IL-17 on endothelial cells for inflammatory cell recruitment during the inflammation process.

Neutrophils are inflammatory cells believed to be the first-line defenders against pathogens. Neutrophil recruitment and adhesion to endothelial cells has been investigated in inflammatory diseases such as RA, COPD, and cancer [44]. One essential role of endothelial cells during the inflammatory process is coordinating the recruitment of inflammatory cells, including neutrophils, monocytes, and lymphocytes into underlying tissues, and actively participating in neovascularization processes [45, 46]. Previous results have identified IL17 as an important mediator of neutrophilic inflammation [47]. However, neutrophils do not respond directly to IL-17, which suggests that other structural cells, such as endothelial and epithelial cells, might be involved in IL-17-mediated neutrophil recruitment. Although previous reports have indicated that IL-17 can promote the activity of epithelial cells and fibroblasts, and induce smooth muscle cells to recruit neutrophils, the effect and relative mechanism of IL-17 on endothelial cells remains poorly investigated. Our studies reveal that IL-17 activated endothelial cells through STAT3 regulation, promoted the induction of GRO- $\alpha$, GM-CSF, and IL-8, and augmented the recruitment and accumulation of neutrophils. The requirement for STAT3 was established through the use of FLLL31, which is a specific inhibitor of STAT3. Furthermore, siRNA silencing of STAT3 significantly inhibited the induction of inflammatory factors. Therefore, this study reveals a molecular mechanism by which IL-17 mediates neutrophil recruitment through activating endothelial cells via the STAT3 pathway. 


\section{Cellular Physiology Cell Physiol Biochem 2015;36:2340-2356 \begin{tabular}{l|l} 
and Biochemistry Published online: July 27, 2015 & $\begin{array}{l}\text { C 2015 S. Karger AG, Basel } \\
\text { www.karger.com/cpb }\end{array}$ \\
\hline
\end{tabular} \\ Yuan et al.: IL-17 Stimulates STAT3-Mediated Endothelial Cell Activation}

Multiple inflammation regulation pathways, including the PI3K/Akt, Act1/TRAF6/ $N F \kappa B$, and MAPK/ERK signaling pathways have all been associated with IL-17-mediated downstream signaling pathways in different kinds of cells [2]. The induction of these various signaling cascades might be cell-type specific, IL-17 concentration-dependent, or associated with particular target genes. Moreover, since IL-17 can bind IL-17 receptor complexes as a homo- or hetero-dimer, the downstream signaling cascades may be regulated through IL-17RA and/or IL-17RC [8, 23, 48]. Herein, we demonstrate that IL-17 promotes the phosphorylation and nuclear translocation of STAT3 in HMECs. The IL-17-induced activation of STAT 3 could be linked to the release of GRO- $\alpha$, GM-CSF, and IL-8. Activation of STAT3 might function as the downstream regulator of IL-17RA, since we also observed that silencing IL17RA could inhibit the tyrosine phosphorylation of STAT3 and reduce the expression of GRO- $\alpha$, GM-CSF, and IL-8. Importantly, the expression levels of IL-8 and GM-CSF were directly attenuated upon STAT3 inhibition and siRNA silencing, which indicated that the release of these pro-inflammatory cytokines may be directly mediated by STAT3, or indirectly via other transcription factors such as NFKB or C/EBP $\beta$ [49]. However, expression levels of GRO- $\alpha$ were only partly inhibited under the same conditions. These data imply that the regulation of GRO- $\alpha$ may be induced by alternative molecular mechanisms. Together, these data suggest that STAT3 downstream of IL-17RA may be a major mediator of IL-17-mediated activation of endothelial cells. Further investigations on the role of nuclei-associated STAT3 in endothelial cells will provide the detailed mechanism of the regulation of inflammatory factors and chemokine production.

Differences in expression levels of IL-17 receptors, stimulated concentrations of IL17 , and treatment times in endothelial cells may all contribute to the signaling cascades involved. Although we did not observe significant attenuation of IL-17-induced endothelial cell proliferation in the presence of ERK or PI3K inhibitors, respectively (Data not shown), our observations have not excluded the potential involvement of these signaling pathways. For example, the activation of STAT3 can be regulated through the ERK and PI3K pathways [29]. Our data reveal that the phosphorylation of ERK and p38 were also inhibited after silencing of IL-17RA. ERK might play roles in regulation of STAT3 tyrosine phosphorylation in endothelial cells through an alternative mechanism or through other receptors of IL-17. Interestingly, we observed that STAT3 transcriptional activity was blocked by PI3K inhibition. Since STAT3 is a downstream regulator of PI3K $[48,50]$, and IL-17 has been reported to activate endothelial cells through PI3K, our observations therefore elucidate the potential role of STAT3 in the regulation of IL-17 in endothelial cells as the mediator of PI3K signals.

FLLL31 is a newly developed STAT3 inhibitor which specifically suppresses the Y705 phosphorylation of STAT3 [34]. Previously, we identified that FLLL31 specifically inhibited STAT3 phosphorylation, attenuated LPS-stimulated NO, and reduced inflammatory factor production in macrophages. In addition, FLLL31 inhibited the proliferation and lymphoblastic transformation of lymphocytes. Moreover, FLLL31 significantly attenuated recruitment of inflammatory cells and mucus hypersecretion in DRA-challenged murine lung sections. Importantly, FLLL31 reduced the expression of IL-17 and Ly-6G/Gr- $1^{+}$cells in DRA-challenged murine lungs [51]. Meanwhile, we observed that FLLL31 reduced the expression of CD31 in the lung sections (Data not shown), which is consistent with the observations that IL-17 drives vascular inflammation, endothelial dysfunction, and arterial hypertension in psoriasis-like skin disease in K14-IL-17A(ind/+) mice [18]. Taken together, these data further suggest that inhibition of Y705 on STAT3 could attenuate the endothelial cell activation and recruitment of neutrophils in vivo.

In addition, other mediators of endothelial cell function might be involved in regulating IL-17 signals. HMEC is a JAK3 hyperactivation endothelial cell line, and phosphorylation of JAK3 increased upon IL-17 treatment in HMECs (Data not shown). Therefore, investigations on JAK3 expression and STAT3 regulation might further elucidate the molecular mechanism of IL-17 in HMECs. Moreover, accumulating evidence suggests that the late Y705 phosphorylation of STAT3 is induced by IL- 6 release upon GPCR activation [52]. Activation of GPCRs is frequently linked to the modulation of various transcriptional factors including 


\begin{tabular}{|c|c|c|}
\hline Cellular Physiology & Cell Physiol Biochem 2015;36:2340-2356 & \\
\hline and Biochemistry & \begin{tabular}{l|l|} 
DOI: $10.11159 / 000430197$ \\
Published online: July 27, 2015
\end{tabular} & $\begin{array}{l}\text { O } 2015 \text { S. Karger AG, Basel } \\
\text { www.karger.com/cpb }\end{array}$ \\
\hline
\end{tabular}

STAT3 [53], which plays an important role in hematopoietic development and immune regulation. It is therefore plausible that IL-17 may modulate indirect functions through GPCRmediated STAT3 activation. For example, Gro- $\alpha$ and IL-8 can both promote endothelial cell activation through the chemokine receptor CXCR2 in an autocrine loop [54], and previous investigations have reported that IL-17 promotes angiogenesis through CXCR2 [55]. Thus, the potential autocrine feedback between CXCR2 and IL-17 in mediating STAT3 activation will be an interestingly area of future study.

Overall, this study demonstrates that STAT3 acts as a link between IL-17-mediated endothelial cell activation and neutrophil recruitment, and is important for the release of GRO- $\alpha$, GM-CSF, and IL-8 from endothelial cells stimulated by IL-17.

\section{Abbreviations}

IL-17 (Interleukin-17); HMEC (human dermal microvascular endothelial cells); HL60 (Human promyelocytic leukemia cells); STAT (signal transducers and activators of transcription); GRO- $\alpha$ (Growth-regulated oncogene alpha (CXCL-1)); GM-CSF (Granulocytemacrophage colony-stimulating factor); IL-8 (Interleukin-8 (CXCL-8)); bFGF (basic fibroblast growth factor); TNF- $\alpha$ (Tumor necrosis factor); JAK (Janus kinase); DMEM (Dulbecco's modified Eagle's medium); FCS (Fetal calf serum).

\section{Acknowledgements}

We greatly thank Dr. Hou's lab members for their discussions and suggestions on this manuscript, and Dr. Lin Cui and Dr. Maoyong Song for their technical instruction on immunofluorescent labeling. This work is supported in part by a grant from the National Natural Science Foundation of China (No. 81001442), the Specialized Research Fund for the Doctoral Program of Higher Education (No. 20101106120023), the Central Public-interest Scientific Institution Basal Research Fund (No. 2011CHX04), and Funding from The State Key Laboratory of Environmental Chemistry and Ecotoxicology, RCEES, CAS (KF2012-16).

\section{Disclosure Statement}

The authors declare no conflicts of interest.

\section{References}

1 Korn T, Bettelli E, Oukka M, Kuchroo VK: Il-17 and th17 cells. Annu Rev Immunol 2009;27:485-517.

2 Gu C, Wu L, Li X: Il-17 family: Cytokines, receptors and signaling. Cytokine 2013;64:477-485.

3 Park SJ, Lee YC: Interleukin-17 regulation: An attractive therapeutic approach for asthma. Respir Res 2010;11:78.

4 Torrado E, Cooper AM: Il-17 and th17 cells in tuberculosis. Cytokine Growth Factor Rev 2010;21:455-462.

5 Alcorn JF, Crowe CR, Kolls JK: Th17 cells in asthma and copd. Annu Rev Physiol 2010;72:495-516.

6 Numasaki M, Fukushi J, Ono M, Narula SK, Zavodny PJ, Kudo T, Robbins PD, Tahara H, Lotze MT: Interleukin-17 promotes angiogenesis and tumor growth. Blood 2003;101:2620-2627.

7 Roussel L, Houle F, Chan C, Yao Y, Berube J, Olivenstein R, Martin JG, Huot J, Hamid Q Ferri L, Rousseau S: Il-17 promotes p38 mapk-dependent endothelial activation enhancing neutrophil recruitment to sites of inflammation. J Immunol 2010;184:4531-4537.

8 Gaffen SL: Recent advances in the il-17 cytokine family. Curr Opin Immunol 2011;23:613-619.

9 Pelletier M, Maggi L, Micheletti A, Lazzeri E, Tamassia N, Costantini C, Cosmi L, Lunardi C, Annunziato F, Romagnani S, Cassatella MA: Evidence for a cross-talk between human neutrophils and th17 cells. Blood 2010;115:335-343.

10 Chung AS, Wu X, Zhuang G, Ngu H, Kasman I, Zhang J, Vernes JM, Jiang Z, Meng YG, Peale FV, Ouyang W, Ferrara N: An interleukin-17-mediated paracrine network promotes tumor resistance to anti-angiogenic therapy. Nat Med 2013;19:1114-1123. 


\section{Cellular Physiology Cell Physiol Biochem 2015;36:2340-2356 \begin{tabular}{ll|l} 
and Biochemistry & $\begin{array}{l}\text { DOI: 10.1159/000430197 } \\
\text { Published online: July 27, } 2015\end{array}$ & $\begin{array}{l}\text { C 2015 S. Karger AG, Basel } \\
\text { www.karger.com/cpb }\end{array}$ \\
\hline
\end{tabular} \\ Yuan et al.: IL-17 Stimulates STAT3-Mediated Endothelial Cell Activation}

11 Linden A, Laan M, Anderson GP: Neutrophils, interleukin-17a and lung disease. Eur Respir J 2005;25:159172.

12 Ogura H, Murakami M, Okuyama Y, Tsuruoka M, Kitabayashi C, Kanamoto M, Nishihara M, Iwakura Y, Hirano T: Interleukin-17 promotes autoimmunity by triggering a positive-feedback loop via interleukin-6 induction. Immunity 2008;29:628-636.

13 Brackett CM, Muhitch JB, Evans SS, Gollnick SO: Il-17 promotes neutrophil entry into tumor-draining lymph nodes following induction of sterile inflammation. J Immunol 2013;191:4348-4357.

14 Hosokawa Y, Hosokawa I, Ozaki K, Nakanishi T, Nakae H, Matsuo T: Catechins inhibit ccl20 production in il-17a-stimulated human gingival fibroblasts. Cell Physiol Biochem 2009;24:391-396.

15 Amulic B, Cazalet C, Hayes GL, Metzler KD, Zychlinsky A: Neutrophil function: From mechanisms to disease. Annu Rev Immunol 2012;30:459-489.

16 Walters EH, Reid D, Soltani A, Ward C: Angiogenesis: A potentially critical part of remodelling in chronic airway diseases? Pharmacol Ther 2008;118:128-137.

17 Bailey SR, Boustany S, Burgess JK, Hirst SJ, Sharma HS, Simcock DE, Suravaram PR, Weckmann M: Airway vascular reactivity and vascularisation in human chronic airway disease. Pulm Pharmacol Ther 2009;22:417-425.

18 Karbach S, Croxford AL, Oelze M, Schuler R, Minwegen D, Wegner J, Koukes L, Yogev N, Nikolaev A, Reissig S, Ullmann A, Knorr M, Waldner M, Neurath MF, Li H, Wu Z, Brochhausen C, Scheller J, Rose-John S, Piotrowski C, Bechmann I, Radsak M, Wild P, Daiber A, von Stebut E, Wenzel P, Waisman A, Munzel T: Interleukin 17 drives vascular inflammation, endothelial dysfunction, and arterial hypertension in psoriasis-like skin disease. Arterioscler Thromb Vasc Biol 2014;34:2658-2668.

19 Honorati MC, Neri S, Cattini L, Facchini A: Interleukin-17, a regulator of angiogenic factor release by synovial fibroblasts. Osteoarthritis Cartilage 2006;14:345-352.

20 Chauhan SK, Jin Y, Goyal S, Lee HS, Fuchsluger TA, Lee HK, Dana R: A novel pro-lymphangiogenic function for th17/il-17. Blood 2011;118:4630-4634.

21 Moran EM, Connolly M, Gao W, McCormick J, Fearon U, Veale DJ: Interleukin-17a induction of angiogenesis, cell migration, and cytoskeletal rearrangement. Arthritis Rheum 2011;63:3263-3273.

22 Chen Y, Zhong M, Liang L, Gu F, Peng H: Interleukin-17 induces angiogenesis in human choroidal endothelial cells in vitro. Invest Ophthalmol Vis Sci 2014;55:6968-6975.

23 Tsai HC, Velichko S, Hung LY, Wu R: Il-17a and th17 cells in lung inflammation: An update on the role of th17 cell differentiation and il-17r signaling in host defense against infection. Clin Dev Immunol 2013;2013:267971.

24 Chang SH, Dong C: Signaling of interleukin-17 family cytokines in immunity and inflammation. Cell Signal 2011;23:1069-1075.

25 Chen J, Liao MY, Gao XL, Zhong Q, Tang TT, Yu X, Liao YH, Cheng X: Il-17a induces pro-inflammatory cytokines production in macrophages via mapkinases, nf-kappab and ap-1. Cell Physiol Biochem 2013;32:1265-1274.

26 May MJ: Il-17r signaling: New players get in on the act1. Nat Immunol 2011;12:813-815.

27 Huang F, Kao CY, Wachi S, Thai P, Ryu J, Wu R: Requirement for both jak-mediated pi3k signaling and act1/ traf6/tak1-dependent nf-kappab activation by il-17a in enhancing cytokine expression in human airway epithelial cells. J Immunol 2007;179:6504-6513.

28 Chen Y, Thai P, Zhao YH, Ho YS, DeSouza MM, Wu R: Stimulation of airway mucin gene expression by interleukin (il)-17 through il-6 paracrine/autocrine loop. J Biol Chem 2003;278:17036-17043.

29 Gao H, Ward PA: Stat3 and suppressor of cytokine signaling 3: Potential targets in lung inflammatory responses. Expert Opin Ther Targets 2007;11:869-880.

30 Yang XO, Panopoulos AD, Nurieva R, Chang SH, Wang D, Watowich SS, Dong C: Stat3 regulates cytokinemediated generation of inflammatory helper t cells. J Biol Chem 2007;282:9358-9363.

31 Benekli M, Baer MR, Baumann H, Wetzler M: Signal transducer and activator of transcription proteins in leukemias. Blood 2003;101:2940-2954.

32 Yeh M, Gharavi NM, Choi J, Hsieh X, Reed E, Mouillesseaux KP, Cole AL, Reddy ST, Berliner JA: Oxidized phospholipids increase interleukin 8 (il-8) synthesis by activation of the c-src/signal transducers and activators of transcription (stat)3 pathway. J Biol Chem 2004;279:30175-30181.

33 Yahata Y, Shirakata Y, Tokumaru S, Yamasaki K, Sayama K, Hanakawa Y, Detmar M, Hashimoto K: Nuclear translocation of phosphorylated stat3 is essential for vascular endothelial growth factor-induced human 


\section{Cellular Physiology Cell Physiol Biochem 2015;36:2340-2356

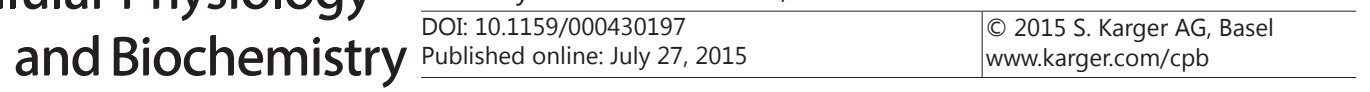 \\ Yuan et al.: IL-17 Stimulates STAT3-Mediated Endothelial Cell Activation}

dermal microvascular endothelial cell migration and tube formation. J Biol Chem 2003;278:40026-40031.

34 Lin L, Hutzen B, Zuo M, Ball S, Deangelis S, Foust E, Pandit B, Ihnat MA, Shenoy SS, Kulp S, Li PK, Li C, Fuchs J, Lin J: Novel stat3 phosphorylation inhibitors exhibit potent growth-suppressive activity in pancreatic and breast cancer cells. Cancer Res 2010;70:2445-2454.

35 Yuan S, Fu Y, Wang X, Shi H, Huang Y, Song X, Li L, Song N, Luo Y: Voltage-dependent anion channel 1 is involved in endostatin-induced endothelial cell apoptosis. Faseb J 2008;22:2809-2820.

36 Zha X, Hu Z, He S, Wang F, Shen H, Zhang H: Tsc1/tsc2 inactivation inhibits akt through mtorc1-dependent up-regulation of stat3-pten cascade. Cancer Lett 2011;313:211-217.

37 Guo Y, Liao Y, Jia C, Ren J, Wang J, Li T: Microrna-182 promotes tumor cell growth by targeting transcription elongation factor a-like 7 in endometrial carcinoma. Cell Physiol Biochem 2013;32:581-590.

38 Jiang HL, Xu D, Yu H, Ma X, Lin GF, Ma DY, Jin JZ: Dax-1 inhibits hepatocellular carcinoma proliferation by inhibiting beta-catenin transcriptional activity. Cell Physiol Biochem 2014;34:734-742.

39 Hauert AB, Martinelli S, Marone C, Niggli V: Differentiated hl-60 cells are a valid model system for the analysis of human neutrophil migration and chemotaxis. Int J Biochem Cell Biol 2002;34:838-854.

40 Lau WW, Ng JK, Lee MM, Chan AS, Wong YH: Interleukin-6 autocrine signaling mediates melatonin mt(1/2) receptor-induced stat3 tyr(705) phosphorylation. J Pineal Res 2012;52:477-489.

41 Hot A, Lenief V, Miossec P: Combination of il-17 and tnfalpha induces a pro-inflammatory, pro-coagulant and pro-thrombotic phenotype in human endothelial cells. Ann Rheum Dis 2012;71:768-776.

42 Fujie H, Niu K, Ohba M, Tomioka Y, Kitazawa H, Nagashima K, Ohrui T, Numasaki M: A distinct regulatory role of th17 cytokines il-17a and il-17f in chemokine secretion from lung microvascular endothelial cells. Inflammation 2012;35:1119-1131.

43 Pickens SR, Volin MV, Mandelin AM 2nd, Kolls JK, Pope RM, Shahrara S: Il-17 contributes to angiogenesis in rheumatoid arthritis. J Immunol 2010;184:3233-3241.

44 Mantovani A, Cassatella MA, Costantini C, Jaillon S: Neutrophils in the activation and regulation of innate and adaptive immunity. Nat Rev Immunol 2011;11:519-531.

45 Kolaczkowska E, Kubes P: Neutrophil recruitment and function in health and inflammation. Nat Rev Immunol 2013;13:159-175.

46 Williams MR, Azcutia V, Newton G, Alcaide P, Luscinskas FW: Emerging mechanisms of neutrophil recruitment across endothelium. Trends Immunol 2011;32:461-469.

47 Laan M, Cui ZH, Hoshino H, Lotvall J, Sjostrand M, Gruenert DC, Skoogh BE, Linden A: Neutrophil recruitment by human il-17 via c-X-c chemokine release in the airways. J Immunol 1999;162:2347-2352.

48 Gu FM, Li QL, Gao Q, Jiang JH, Zhu K, Huang XY, Pan JF, Yan J, Hu JH, Wang Z, Dai Z, Fan J, Zhou J: Il-17 induces akt-dependent il-6/jak2/stat3 activation and tumor progression in hepatocellular carcinoma. Mol Cancer 2011;10:150.

49 Sun D, Novotny M, Bulek K, Liu C, Li X, Hamilton T: Treatment with il-17 prolongs the half-life of chemokine cxcl1 mrna via the adaptor traf5 and the splicing-regulatory factor sf2 (asf). Nat Immunol 2011;12:853860.

50 Stalinska K, Guzdek A, Rokicki M, Koj A: Transcription factors as targets of the anti-inflammatory treatment. A cell culture study with extracts from some mediterranean diet plants. J Physiol Pharmacol 2005;56:157-169.

51 Yuan S, Cao S, Jiang R, Liu R, Bai J, Hou Q: Flll31, a derivative of curcumin, attenuates airway inflammation in a multi-allergen challenged mouse model. Int Immunopharmacol 2014;21:128-136.

52 Sano M, Fukuda K, Kodama H, Takahashi T, Kato T, Hakuno D, Sato T, Manabe T, Tahara S, Ogawa S: Autocrine/paracrine secretion of il-6 family cytokines causes angiotensin ii-induced delayed stat3 activation. Biochem Biophys Res Commun 2000;269:798-802.

53 Yuen JW, Poon LS, Chan AS, Yu FW, Lo RK, Wong YH: Activation of stat3 by specific Galpha subunits and multiple gbetagamma dimers. Int J Biochem Cell Biol 2010;42:1052-1059.

54 Strieter RM, Burdick MD, Gomperts BN, Belperio JA, Keane MP: Cxc chemokines in angiogenesis. Cytokine Growth Factor Rev 2005;16:593-609.

55 Numasaki M, Watanabe M, Suzuki T, Takahashi H, Nakamura A, McAllister F, Hishinuma T, Goto J, Lotze MT, Kolls JK, Sasaki H: Il-17 enhances the net angiogenic activity and in vivo growth of human non-small cell lung cancer in scid mice through promoting cxcr-2-dependent angiogenesis. J Immunol 2005;175:61776189. 